\title{
Common genetic variation in and near the melanocortin 4 receptor gene $(M C 4 R)$ is associated with body mass index in American Indian adults and children
}

\author{
Yunhua L. Muller $\cdot$ Marie S. Thearle $\cdot$ Paolo Piaggi $\cdot$ Robert L. Hanson $\cdot$ \\ Duncan Hoffman • Brittany Gene • Darin Mahkee - Ke Huang • Sayuko Kobes • \\ Susanne Votruba $\cdot$ William C. Knowler $\cdot$ Clifton Bogardus $\cdot$ Leslie J. Baier
}

Received: 11 June 2014 / Accepted: 1 August 2014 / Published online: 8 August 2014

(C) The Author(s) 2014. This article is published with open access at Springerlink.com

\begin{abstract}
Six rare functional coding mutations were previously identified in melanocortin 4 receptor $(M C 4 R)$ in 6,760 American Indians. Individuals heterozygous for one of these mutations become obese while young. We now investigate whether common non-coding variation near $M C 4 R$ also contributes to obesity. Fifty-six tag single-nucleotide polymorphisms (SNPs) were genotyped in 3,229 full-heritage Pima Indians, and nine of these SNPs which showed evidence for association were genotyped in additional 3,852 mixed-heritage American Indians. Associations of SNPs with maximum body mass index (BMI) in adulthood $(n=5,918)$, BMI $z$ score in childhood $(n=5,350)$, percent body fat $(n=864)$, energy expenditure $(n=358)$ and ad libitum food intake $(n=178)$ were assessed. Conditional analyses demonstrated that SNPs, rs74861148 and rs483125, were independently associated with BMI in adulthood $\left(\beta=0.68 \mathrm{~kg} / \mathrm{m}^{2}\right.$ per risk allele, $p=5 \times 10^{-5} ; \beta=0.58 \mathrm{~kg} / \mathrm{m}^{2}, p=0.002$, respectively) and BMI $z$ score in childhood ( $\beta=0.05, p=0.02$; $\beta=0.07, p=0.01$, respectively). One haplotype (frequency $=0.35$ ) of the $\mathrm{G}$ allele at rs74861148 and the A allele at rs 483125 provided the strongest evidence for association
\end{abstract}

Electronic supplementary material The online version of this article (doi:10.1007/s00439-014-1477-6) contains supplementary material, which is available to authorized users.

Y. L. Muller · M. S. Thearle · P. Piaggi · R. L. Hanson ·

D. Hoffman $\cdot$ B. Gene $\cdot$ D. Mahkee $\cdot$ K. Huang $\cdot$ S. Kobes $~$

S. Votruba · W. C. Knowler · C. Bogardus · L. J. Baier $(\bowtie)$

Phoenix Epidemiology and Clinical Research Branch, National Institute of Diabetes and Digestive and Kidney Disease, National Institutes of Health, 445 North 5th street, Phoenix, AZ 85004, USA

e-mail: 1baier@phx.niddk.nih.gov

Y. L. Muller

e-mail: ymuller@mail.nih.gov with adult BMI $\left(\beta=0.89 \mathrm{~kg} / \mathrm{m}^{2}, p=5.5 \times 10^{-7}\right)$, and was also associated with childhood BMI $z$ score $(\beta=0.08$, $p=0.001)$. In addition, a promoter SNP rs11872992 was nominally associated with adult BMI $\left(\beta=0.61 \mathrm{~kg} / \mathrm{m}^{2}\right.$, $p=0.05)$ and childhood BMI $z$ score $(\beta=0.11, p=0.01)$, where the risk allele also modestly decreased transcription in vitro by $12 \%(p=0.005)$. This risk allele was further associated with increased percent body fat $(\beta=2.2 \%, p=0.002)$, increased food intake $(\beta=676 \mathrm{kcal} / \mathrm{day}, p=0.007)$ and decreased energy expenditure $(\beta=-53.4 \mathrm{kcal} / \mathrm{day}$, $p=0.054)$. Common and rare variation in $M C 4 R$ contributes to obesity in American Indians.

\section{Introduction}

The melanocortin pathway in the human hypothalamus plays a pivotal role in regulating food intake and energy homeostasis. Melanocortin 4 receptor $(M C 4 R)$ haploinsufficiency is the most common monogenic cause of obesity, with a prevalence of $1.7-3.0 \%$ in obese individuals (Calton et al. 2009; Hainerová et al. 2007; Hinney et al. 2006). We previously identified 10 rare non-synonymous mutations by sequencing the entire coding region of the $M C 4 R$ gene in 6,760 American Indians, predominately of Pima Indian heritage. In vitro luciferase assays showed that six of these mutations had a functional impact on the receptor's activity. The population prevalence of these combined functional coding mutations in $M C 4 R$ was $2.4 \%$, and in these individuals the rate of increase in body mass index (BMI) and risk of type 2 diabetes (T2D) was more apparent during childhood as compared with adulthood (Thearle et al. 2012). Since obesity is very common among American Indians, the high prevalence of this disease cannot be solely explained by these rare $M C 4 R$ 
mutations. Genome-wide association studies (GWASs) in Caucasians and other ethnic groups have identified common non-coding variants near $M C 4 R$ that are reproducibly associated with fat mass, body weight and risk of obesity (Loos et al. 2008; Willer et al. 2009; Speliotes et al. 2010). Several risk alleles in $M C 4 R$, which are common and consistently associated with obesity/BMI in studies of Caucasians (e.g., rs17782313, rs17700633 and rs571312), are uncommon in American Indians (frequency $=0.03$, 0.005 and 0.003 , respectively) despite American Indians having much higher rates of obesity than Caucasians. This raises the possibility that different variants in $M C 4 R$ may contribute to obesity in American Indians. Therefore, in the current study, we investigate the relative contribution of common variation across $M C 4 R$ on risk of obesity in American Indians, and further explore the effect of common $M C 4 R$ variation on energy intake and energy expenditure measures.

\section{Methods}

\section{Subjects}

Subjects are participants of a longitudinal study of the etiology of T2D among the Gila River Indian Community in Arizona, where most of the residents are Pima Indians or Tohono O'odham (a closely related tribe) (Knowler et al. 1990). Individuals participated in biennial examinations that included measurements of BMI, and fasting and 2-h glucose and insulin concentrations during a 75 -g oral glucose tolerance test (OGTT). Diabetes was determined by prior clinical diagnosis or an oral glucose tolerance test according to the criteria of American Diabetes Association (The Expert Committee on the Diagnosis and Classification of Diabetes Mellitus 1997). For analysis of maximum BMI during adulthood, the highest BMI after the age of 15 years recorded from a non-diabetic exam was considered $\left(n=5,918\right.$, males $44 \%$, BMI $35.1 \pm 8.4 \mathrm{~kg} / \mathrm{m}^{2}$, age $29.7 \pm 11.4$ years). For analysis of childhood obesity, the maximum sex- and age-specific BMI $z$ score (Pima based) was identified between the ages of 5 and 20 years ( $n=5,350$, males $45 \%$, BMI $27.2 \pm 6.7 \mathrm{~kg} / \mathrm{m}^{2}$, age $13.8 \pm 3.9$ years). Data including BMI measured at multiple non-diabetic exams (range 1-17 exams for a given individual during adulthood; 1-8 exams during childhood) between the years 1965 and 2004 were also analyzed. The mean BMI from all non-diabetic exams during adulthood was $32.7 \pm 8.0 \mathrm{~kg} / \mathrm{m}^{2}$, and the mean BMI during childhood was $25.5 \pm 6.2 \mathrm{~kg} / \mathrm{m}^{2}$ ( $z$ scores were converted to BMI units using the mean and standard deviation of 12-year-old Pima females). Among these subjects, 3,229 were full-heritage Pima Indian; the remaining 3,852 subjects were mixed heritage, on average one-half Pima and three-quarters American Indian. DNA from all of these American Indians had been previously sequenced for $M C 4 R$ coding mutations, and the 167 individuals had a rare functional coding mutation. With the severe functional impairment caused by the rare coding variants in these individuals (BMI $39.0 \pm 7.6 \mathrm{~kg} / \mathrm{m}^{2}$ in adulthood, $32.7 \pm 8.0 \mathrm{~kg} / \mathrm{m}^{2}$ in childhood) (Thearle et al. 2012), the additional influence of any, presumably more moderate, functional effects of common variants is likely negligible. Therefore, these 167 individuals were excluded from the present study.

Quantitative metabolic traits and ad libitum food intake study

Among the subjects who were genotyped, 538 non-diabetic American Indians (predominately full-heritage Pima Indians, males $58 \%$, age $27 \pm 6$ years, BMI $34 \pm 8 \mathrm{~kg} / \mathrm{m}^{2}$ ) had undergone detailed metabolic studies in our Clinical Research Unit (CRU). Body composition was estimated by underwater weighing until 1994 and by dual energy X-ray absorptiometry (DPX-1, Lunar Radiation Corp.) thereafter (Tataranni and Ravussin 1995). Energy expenditure and sleeping metabolic rate over $24 \mathrm{~h}$ were assessed in 358 non-diabetic subjects using whole-room indirect calorimetry as previously described (Ravussin et al. 1986). Spontaneous physical activity (SPA) was detected by radar sensors and expressed as percentage of time in motion over $24 \mathrm{~h}$. Sleeping metabolic rate was defined as the average energy expenditure between 01:00 am and 05:00 am during which SPA was $<1.5 \%$ (Piaggi et al. 2013a) and the thermic effect of the last meal was undetected (Piaggi et al. 2013b). As a confirmatory measure, resting metabolic rate was assessed by indirect calorimetry using a ventilated hood system before the hyperinsulinemic, euglycemic clamp as previously described (Lillioja et al. 1993; Bogardus et al. 1986).

Also genotyped were a subset of adults who had participated in an ad libitum food intake study as previously described (Pannacciulli et al. 2007). Among 178 participants who had both genotype and food intake data (males $60 \%$, age $34.7 \pm 9.0$ years, BMI $32.7 \pm 8.0 \mathrm{~kg} / \mathrm{m}^{2}$ ), $34 \%$ were full-heritage Pima Indians, $41 \%$ were mixed-heritage American Indians, and $25 \%$ were Caucasians. Food intake was also assessed in a separate study of the relationship between in utero exposures and maternal environmental influences on food intake in full-heritage Pima Indian children. Fifty-five of them had genotype and food intake data (males $49 \%$, age $9.5 \pm 1.2$ years, BMI $25.4 \pm 6.7 \mathrm{~kg} / \mathrm{m}^{2}$ ) (Gluck et al. 2009). Ad libitum food intake in both studies was assessed over 3 days by an automated vending machine system in our CRU (Pannacciulli et al. 2007). 
Selection and genotyping of $M C 4 R$ tag SNPs

Common variation (minor allele frequency, $\mathrm{mAF} \geq 0.01$ ) across a $\sim 510 \mathrm{~kb}$ region encompassing $M C 4 R$ (chr18:57778138-58288450, $\sim 250 \mathrm{~kb}$ flanking each side of the gene, GRCh37/hg19) was identified from wholegenome sequence data $(30-40 \times$ coverage) of 234 Pima Indians (Complete Genomics Inc., Mountain View, CA; Illumina, San Diego, CA, USA) (Muller et al. 2014). Linkage Disequilibrium (LD) between variants was determined using Haploview (version 4.2). Tag SNPs were selected using the Tagger algorithm with a pair-wise $r^{2} \geq 0.8$. SNPs were genotyped using the TaqMan Open Array System (Applied Biosystems, Carlsbad, CA, USA) or BeadXpress System (Illumina, San Diego, CA, USA).

\section{Statistical analyses}

Statistical analyses were performed using the software of the SAS Institute (Cary, NC, USA). The association of quantitative traits including maximum BMI, maximum childhood BMI $z$ score and metabolic traits with genotypes was analyzed by linear regression using the generalized estimating equation procedure (GEE) to account for family membership (sibship). $p$ values were adjusted for multiple covariates as indicated. The individual estimate of the proportion of European ancestry was derived by the method of Hanis et al. (1986) from 45 informative markers with large differences in allele frequency between populations (Tian et al. 2007) for use as a covariate in all analyses. The association between genotype and adult BMI or childhood $z$ score was also analyzed using all longitudinal non-diabetic BMI measurements from an individual. A linear mixed model (PROC MIXED) was fitted that included genotype as a fixed effect along with age, sex, birth year and heritage as covariates. The model also included random effects representing sibship and individual (to account for multiple examinations within an individual). To model the relationship between multiple examinations within an individual, an autoregressive correlation structure was used as previously described (Ma et al. 2010). The difference in change in BMI over time between genotypes was assessed with mixed models to account for repeated measures using a compound symmetry covariance structure and modeling BMI change over time as a linear function for simplicity. The general association of genotype with T2D was assessed with logistic regression analysis and adjusted for covariates, which included age, sex, birth year and heritage. The model was fitted with GEE to account for correlation among siblings.

Conditional analyses were also conducted in which a SNP of interest was added to a model containing one or more additional SNPs. Haplotypes were determined by a modification of the zero recombinant haplotyping method as previously described (Vozarova de Courten et al. 2005). The MLINK program is used to calculate the probability that each individual carries one or two copies of each haplotype, given their genotypes and the genotypes of their family members. These probabilities were then analyzed in regression models in a fashion analogous to individual SNPs.

Functional analysis of the promoter activity of the $M C 4 R$ SNP

A 634-bp DNA fragment of the $M C 4 R$ promoter region containing either an $A$ or $G$ nucleotide at position chr18:58040587 for rs11872992 was amplified with primers forward $5^{\prime}$-ATCTCGAGTGTTAGGGGCTGTA- $3^{\prime}$ and reverse $5^{\prime}$-TCAGGTACCAATAGAGAAATATGG-3' (XhoI and $K p n I$ restriction sites are underlined, respectively). PCR products were inserted at $\mathrm{KpnI}$ and $\mathrm{XhoI}$ sites upstream of the pGL3-Basic luciferase reporter vector (Promega, Madison, WI, USA).

Transient transfection in the murine N-42 hypothalamus cell line (Cellutions Biosystems, Inc., Burlington, ON, Canada) and dual luciferase assays (Promega, Madison, WI, USA) were performed using the modified method as previously described (Thearle et al. 2012; Muller et al. 2003). Four separate transfections were performed and each transfection was repeated in triplicate. The statistical difference in mean luciferase activity between the $\mathrm{G}$ and $\mathrm{A}$ allele at rs11872992 was analyzed by an unpaired $t$ test.

\section{Results}

Association of $M C 4 R$ tag SNPs with adult BMI and childhood BMI $z$ score

SNPs $(n=56)$ tagging common variation $(\mathrm{mAF} \geq 0.01)$ in Pima Indians across a $\sim 510 \mathrm{~kb}$ region encompassing $M C 4 R$ (Supplemental Figure) were initially genotyped in full-heritage Pima subjects from the Gila River Indian Community $(n=3,229$, Supplementary Table 1$)$. SNPs that showed a trend for association (7 SNPs with $p \leq 0.01$ ) with either maximum BMI in adulthood or maximum BMI $z$ score in childhood, or were predicted to be damaging using the Ingenuity Variant Analysis (rs11872992) were further genotyped in all remaining subjects $(n=3,852$, mixed-heritage American Indians, Supplementary Table 1). In addition, rs 17782313 which is the highly replicated common SNP near MC4R in other populations (Loos et al. 2008; Willer et al. 2009; Speliotes et al. 2010) was also genotyped in all study subjects. These nine SNPs were analyzed for associations with maximum BMI in adulthood $(n=5,918)$ 


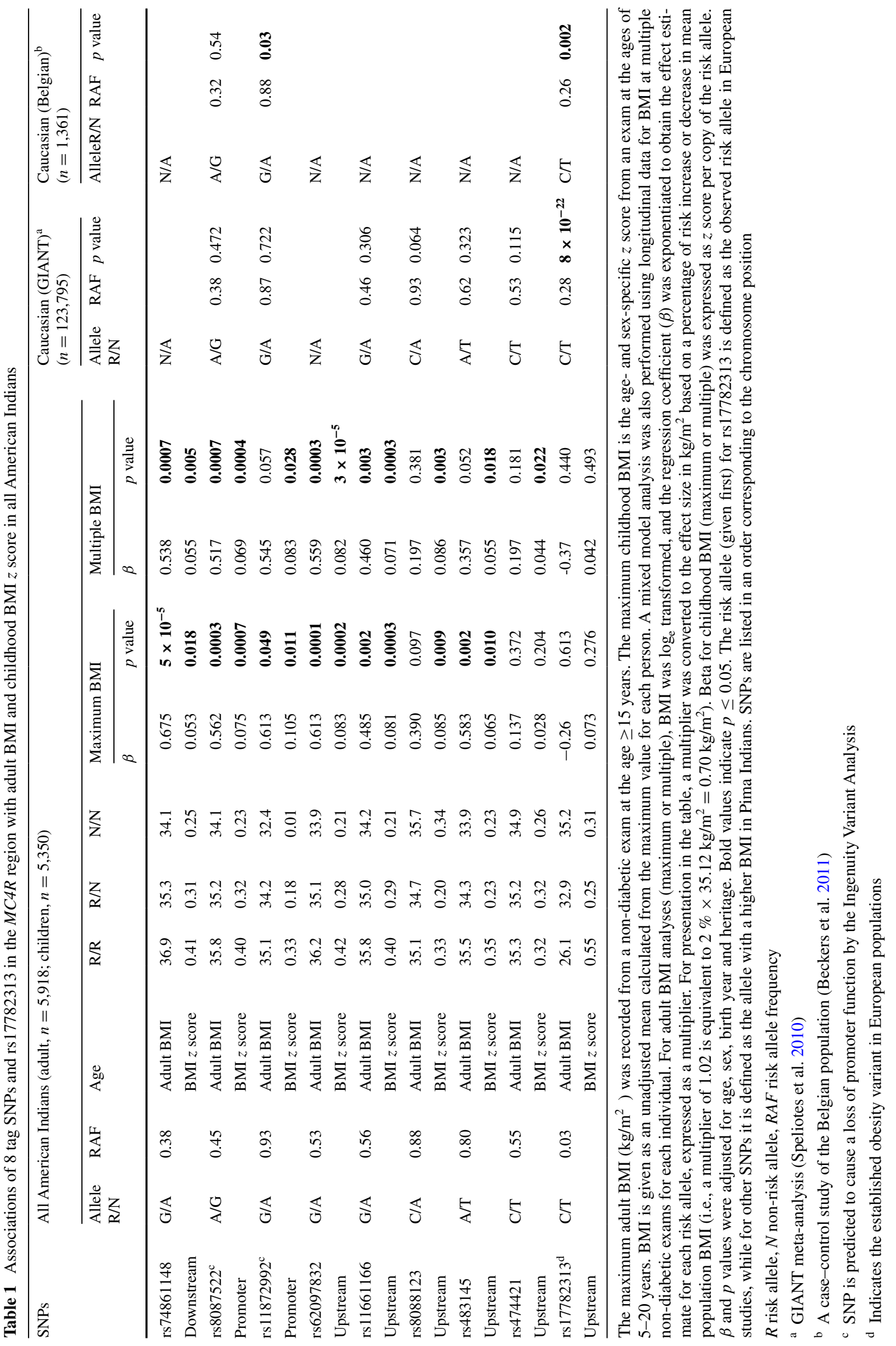


Fig. 1 Additive effects on maximum BMI in American Indian adults (a) and maximum BMI $z$ score in children (b) with increasing numbers of risk alleles of rs74861148 and rs 483154 . Only $3.3 \%$ subjects carried non-risk alleles at both loci (\#risk allele $=0$ ), thus these subjects were combined with those who carried one risk allele from either locus (\#risk allele $=1$ ). Maximum BMI values (mean $\pm \mathrm{SE}$ ), allelic effects $(95 \% \mathrm{CI})$ and $p$ values were adjusted for age, sex, birth year and heritage

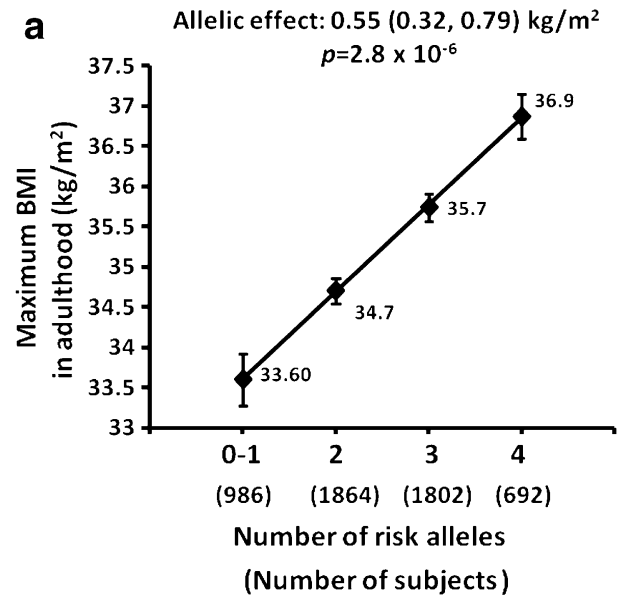

and maximum BMI $z$ score in childhood $(n=5,350)$ in all American Indians as shown in Table 1. A mixed model analysis was also performed using longitudinal data for BMI at multiple exams for each individual, and statistical results were comparable with association data for maximum BMI (Table 1). For further comparison, association data for BMI from the large GIANT (Genetic Investigation of ANthropometric Traits) meta-analysis of Caucasians (Speliotes et al. 2010) and a case-control study of the Belgian population (Beckers et al. 2011) are also shown in Table 1.

The strongest single SNP association with maximum BMI in American Indian adults was observed for rs74861148 with a risk allele frequency (RAF) of 0.38 , mapping $220 \mathrm{~kb}$ downstream of MC4R. Rs74861148 was associated with maximum BMI in 5,918 adults $[\beta=0.68$ $(95 \%$ CI $0.35,1.01) \mathrm{kg} / \mathrm{m}^{2}$ per risk allele, $p=5 \times 10^{-5}$, adjusted for age, sex, birth year and heritage]. Individuals who were homozygous for the risk allele (GG) had an increased adjusted BMI of $1.4 \mathrm{~kg} / \mathrm{m}^{2}$ compared with those with the non-risk allele (AA). This SNP was also nominally associated with maximum BMI $z$ score in 5,350 children $[\beta=0.05(0.01,0.10)$, adjusted $p=0.02]$. In Caucasian and African populations, this SNP is monomorphic for the $\mathrm{G}$ (risk) allele.

Single marker analyses identified weaker evidence for association between maximum BMI in adulthood and six other tag SNPs: rs8087522, rs11872992, rs62097832, rs11661166, rs8088123 and rs483145 (adjusted $p \leq 0.05$, Table 1). All tag SNPs showing any evidence for association were in high $D^{\prime}(0.52-1.00)$, although in low $r^{2}$ (0.01-0.67). To determine the extent to which rs 75861148 contributed to each of these other associations, conditional analyses were conducted in a step-wise fashion. Controlled for rs 74861148 genotypes, only rs483145 (mapping $\sim 128 \mathrm{~kb}$ upstream of $M C 4 R$ with RAF $=0.80$ ) remained associated with maximum BMI (conditional $p=0.02$ ).
When both rs 74861148 and rs 483145 were included in the model, none of other SNPs remained associated with BMI (all conditional $p>0.05$ ), suggesting that both rs74861148 and rs483145 ( $\left.D^{\prime}=0.58, r^{2}=0.06\right)$ independently contribute to the BMI associations in American Indians. However, rs483145 was not associated with BMI in the GIANT meta-analysis $(p=0.32$; Table 1$)$.

To quantify the additive effects of rs74861148 and rs483145 on maximum BMI in American Indians, we analyzed BMI of individuals according to the number of risk alleles at these SNPs. Each additional risk allele increased BMI by $0.55(0.32,0.79) \mathrm{kg} / \mathrm{m}^{2}$ in adulthood (Fig. 1a, $p$ for trend $=2.8 \times 10^{-6}$, adjusted for age, sex, birth year and heritage). Among 5,918 subjects, only $3.3 \%$ were homozygous for non-risk alleles at both loci, and there was no difference in BMI $(p>0.05)$ between this group and those who carry one of the four risk alleles, hence these two groups were combined (\#risk allele $=0-1)$. Individuals with four risk alleles $(12.9 \%$ of the population) had the highest adjusted BMI compared with those with 0 or 1 risk allele $(18.5 \%$ of the population, adjusted $\triangle \mathrm{BMI}=3.3 \mathrm{~kg} / \mathrm{m}^{2}$ ). In children, each risk allele increased BMI $z$ score by $0.05(0.02,0.08)$, corresponding to a BMI increase of $0.30(0.12,0.49) \mathrm{kg} /$ $\mathrm{m}^{2}$ (Fig. $1 \mathrm{~b}$, adjusted $p$ for trend $=0.002$ ). The adjusted $\Delta z$ score between individuals with $0-1$ risk alleles and those with four risk alleles was 0.23 , corresponding to $\Delta$ BMI of $1.4 \mathrm{~kg} / \mathrm{m}^{2}$. Together, the common genotypes of rs 74861148 and rs 483145 accounted for 0.50 and $0.26 \%$ of the variance in liability for adulthood and childhood BMI, respectively.

Haplotype effects of $M C 4 R$ SNPs on maximum BMI and the rate of BMI change

Haplotype analyses for these two independent SNPs rs74861148 (G/A) and rs483145 (A/T) showed that three 
Fig. 2 Haplotype effects of rs74861148 and rs483154 on maximum BMI in American Indian adults (a) and maximum BMI $z$ score in children (b). Haplotype frequency is indicated in the parenthesis. Adult BMI and BMI $z$ score are presented as an adjusted mean \pm SE for each haplotype group. $\beta$ represents the effect per copy of the risk haplotype. $p$ values (additive model) and $\beta(95 \% \mathrm{CI})$ were adjusted for age, sex, birth year and heritage

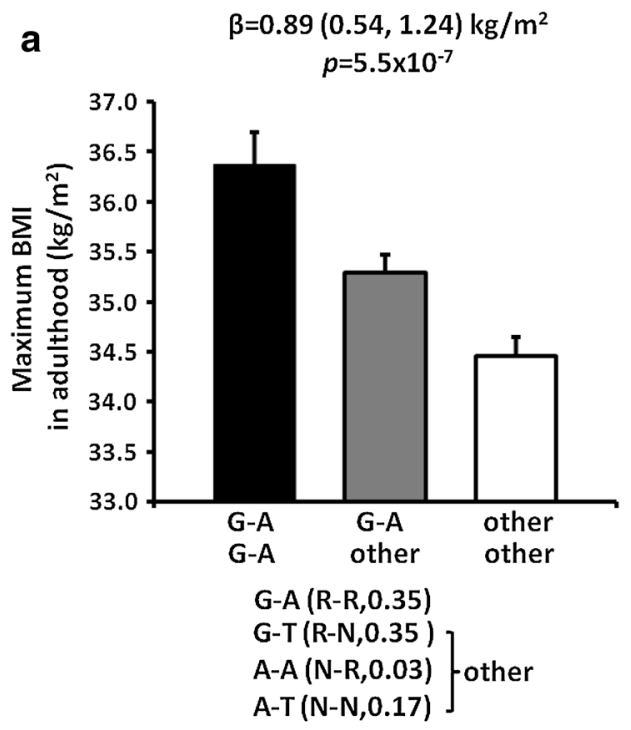

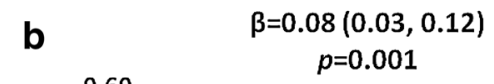

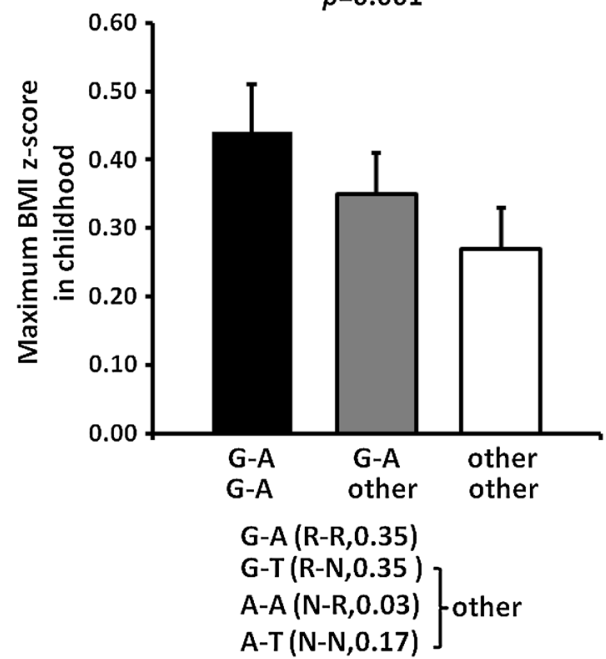

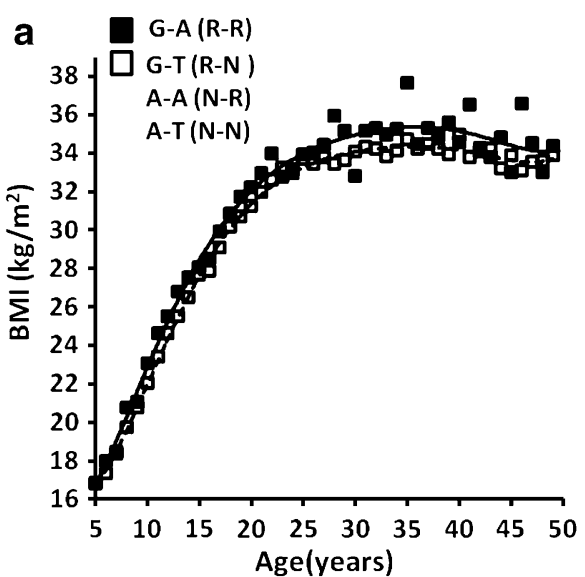

Fig. 3 a Haplotype effects of rs 74861148 and rs483154 on the lifetime BMI trajectory (age 5-50 years). b Haplotype effects of rs74861148 and rs483154 on the rate of BMI change in American Indian children and adults. The risk haplotype carriers (at least one

out of four haplotypes were common in American Indians $(\mathrm{G}-\mathrm{A}$, frequency $=0.35 ; \mathrm{G}-\mathrm{T}$, frequency $=0.03$; $\mathrm{A}-\mathrm{A}$, frequency $=0.44 ; \mathrm{A}-\mathrm{T}$, frequency $=0.17$ ). There were no significant differences in maximum BMI between individuals carrying haplotypes G-T, A-A or A-T. However, individuals with the haplotype $\mathrm{G}-\mathrm{A}$ ( $\mathrm{G}$ risk allele at rs74861148 and A risk allele at rs483145) had a higher maximum BMI as compared with other three haplotypes combined (G-T, A-A and A-T) [Fig. 2a, $\beta=0.89$ $(0.54,1.24) \mathrm{kg} / \mathrm{m}^{2}$ per copy of the G-A haplotype, $p=5.5 \times 10^{-7}$, adjusted for age, sex, birth year and heritage]. In children, those carrying the G-A haplotype also had a higher maximum BMI $z$ score as compared with those carrying all other haplotypes combined [Fig. 2b, $\beta=0.08(0.03,0.12)$ per copy of the $\mathrm{G}-\mathrm{A}$ haplotype,

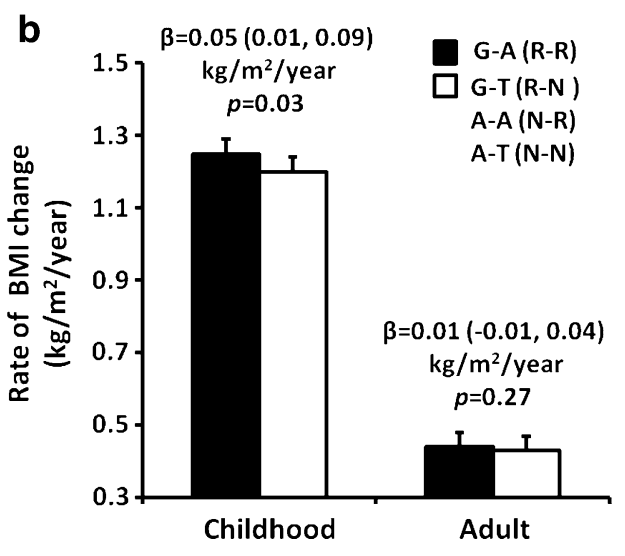

copy) were compared with the non-risk haplotype carriers (no copies). BMI is given as an unadjusted mean at each age group. Rates of BMI change (mean $\pm \mathrm{SE}$ ), beta $(95 \% \mathrm{CI})$ and $p$ values were adjusted for sex, birth year and heritage

corresponding to BMI of $0.49(0.12,0.73) \mathrm{kg} / \mathrm{m}^{2}$, adjusted $p=0.001]$. BMI change over time between the ages of 5-50 years was evaluated in 4,505 American Indians children $(13,168$ total visits, $3.0 \pm 1.5$ visits per subject) and 4,160 adults $(12,582$ total visits, $3.0 \pm 2.3$ visits per subject) with longitudinal BMI measures before developing diabetes. The lifetime BMI trajectory of the risk haplotype (carriers, at least one copy) vs. non-risk haplotype (noncarriers, no copies) is shown in Fig. 3a. Subjects with the risk haplotype G-A had a consistently higher BMI than subjects with the non-risk haplotype at almost every age. In childhood, the rate of BMI change was $1.25 \pm 0.04 \mathrm{~kg} /$ $\mathrm{m}^{2} /$ year in the risk haplotype carriers compared with $1.20 \pm 0.04 \mathrm{~kg} / \mathrm{m}^{2} /$ year in the non-risk haplotype carriers [Fig. $3 \mathrm{~b}, \beta=0.05(0.01,0.09) \mathrm{kg} / \mathrm{m}^{2} /$ year, $p=0.03$, 
adjusted for sex, birth year and heritage, $n=4,505]$. In adulthood, the slope of BMI change stabilized and was no longer greater in the carriers than that in the non-carriers [Fig. 3b, $\beta=0.01(-0.01,0.04) \mathrm{kg} / \mathrm{m}^{2} /$ year, adjusted $p=0.27, n=4,160]$.

\section{Replication of MC4R SNPs in other studies}

The strongest signal for BMI near MC4R in the GIANT meta-analysis is rs 17782313 with a risk allele frequency of $C=0.28$ (Table 1). The $\mathrm{C}$ allele is much less common in American Indians $(\mathrm{AF}=0.03)$ and was not associated with adult BMI or childhood $z$ score (Table 1). Conversely, the tag SNP rs74861148 associated with BMI in American Indians was not informative (monomorphic) in Caucasians. However, a smaller study of $M C 4 R$ in a Belgian case/control study (1,049 obese/312 control) reported that a promoter SNP rs11872992 was associated with obesity $(p=0.03$, Table 1$)$, and this signal was independent from rs 17782313 (Beckers et al. 2011). In our study, this promoter SNP rs11872992 $(\mathrm{RAF}=0.93)$ also had a nominal association with maximum BMI in adulthood [Table 1 , $\left.\beta=0.61(0.01,1.23) \mathrm{kg} / \mathrm{m}^{2}, p=0.05, n=5,918\right)$ and maximum BMI $z$ score in childhood $[\beta=0.11(0.02$, $0.19), p=0.01, n=5,350]$, where the risk allele was consistent with the Belgian study. This variant is located $586 \mathrm{bp}$ from the translational start site of $M C 4 R$, and predicted to cause a loss of promoter function by the Ingenuity Variant Analysis (www.ingenuity.com) by disrupting a putative transcription factor binding site for GATA2. Therefore, although our conditional analyses indicated that the weaker association of rs11872992 with BMI in American Indians could largely be explained by the stronger association of rs74861148 $\left(D^{\prime}=0.69\right)$, we pursued additional studies with this variant.

In vitro functional analyses of promoter activity of rs11872992

The promoter activity of rs11872992 was assessed by an in vitro luciferase assay in a murine hypothalamus cell line (Fig. 4). Overall, the $M C 4 R$ promoter region containing rs11872992 increased luciferase activity by $\sim 18$ fold, as compared with a promoter-less luciferase vector (pGL3-Basic), indicating the strong promoter activity in this region. The DNA construct with the BMI risk allele $\mathrm{G}$ at rs11872992 had a modest, but consistent decrease $[\beta=-12 \%(-20,-4 \%)$ in promoter activity in contrast to the non-risk A allele $(p=0.005)$. SNP rs8087522 was also predicted to cause a loss of promoter function (Ingenuity Variant Analysis); however, our in vitro functional analyses did not detect a difference between the alleles of rs8087522 (data not shown).

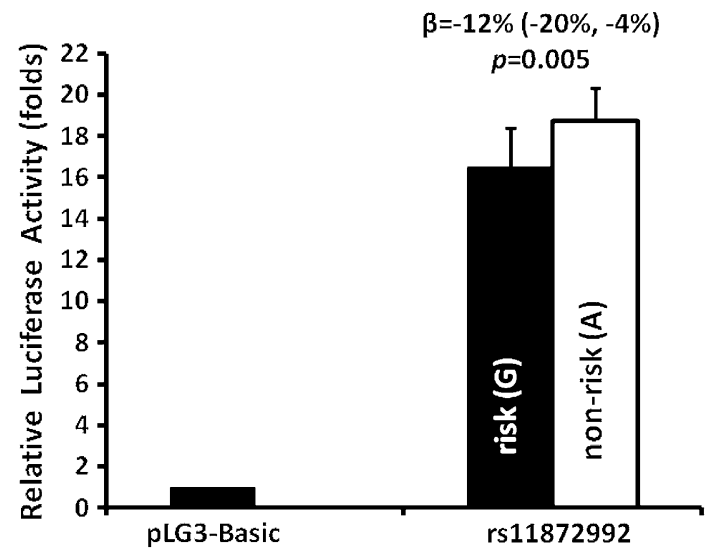

Fig. 4 In vitro functional analyses of promoter activity of rs11872992. Relative luciferase activity (fold change) was expressed as a ratio of firefly luciferase activity to renilla luciferase activity, and further normalized to pGL3-basic luciferase activity. Data are presented as mean $\pm \mathrm{SD}, n=12$ transfections. The statistical difference in the averaged activity was analyzed by an unpaired $t$ test

Additional phenotype analyses of the functional promoter SNP rs11872992

To assess the effect of rs11872992 on longitudinal changes in BMI, we performed mixed model analyses to include data from all available exams and analyzed differences in change in BMI or childhood BMI $z$ score over time. Rs11872292 had a low allele frequency for the A allele $(\mathrm{mAF}=0.07)$, therefore the heterozygous $\mathrm{GA}$ and homozygous AA genotypes were combined for analyses. During childhood, the rate of BMI change was $1.27 \mathrm{~kg} / \mathrm{m}^{2} /$ year in subjects with the risk GG alleles vs. $1.20 \mathrm{~kg} / \mathrm{m}^{2} /$ year in subjects with the non-risk $\mathrm{GA}+\mathrm{AA}$ alleles $[\beta=0.07$ $(0.02,0.11) \mathrm{kg} / \mathrm{m}^{2} /$ year, adjusted $\left.p=0.004\right]$. In adulthood, the accelerated rate of BMI gain continued in individuals with the risk alleles GG compared with those with the nonrisk alleles GA + AA [0.26 vs. $0.22 \mathrm{~kg} / \mathrm{m}^{2} /$ year, $\beta=0.04$ $(0.01,0.07) \mathrm{kg} / \mathrm{m}^{2} /$ year, adjusted $\left.p=0.01\right]$. Many of these subjects $(n=7,667)$ also had data available for T2D status, and rs 11872992 was associated with increased risk for T2D such that individuals with the risk allele for BMI (G allele) had a higher prevalence of T2D with an odds ratio of 1.23 per copy of the risk allele $\mathrm{G}$ (95\% CI 1.03, 1.46, $p=0.02$, adjusted for age, sex, birth year and heritage). However, the association with T2D was attenuated after adjustment for maximum BMI (adjusted $p=0.09$ ), suggesting that the finding was mainly attributable to increased BMI.

A subset of the individuals $(n=864)$ with genotypic data also had detailed measures of metabolic traits related to obesity (Table 2). The risk allele G for BMI at rs11872992 was associated with higher percentage of body fat [Table 2 , $\beta=2.2(0.8,3.6) \%, p=0.002$, adjusted for age, sex and heritage], higher fat mass $[(\beta=3.5(0.9,6.1) \mathrm{kg}$, adjusted 
Table 2 Association of rs11872992 with metabolic traits and ad libitum energy intake in non-diabetic American Indians

\begin{tabular}{|c|c|c|c|c|}
\hline & \multirow[t]{2}{*}{ GG risk/risk } & \multicolumn{3}{|c|}{ rs11872992 (G/A) (mean \pm SD) } \\
\hline & & $\mathrm{GA}+\mathrm{AA}$ risk/non & $\beta(95 \% \mathrm{CI})$ & $p$ value \\
\hline Subjects, body composition $(n)$ & 760 & 104 & & \\
\hline Percentage of body fat & $33.8 \pm 8.4$ & $31.0 \pm 8.7$ & $2.2(0.8,3.6)$ & 0.002 \\
\hline Fat mass $(\mathrm{kg})$ & $33.4 \pm 14.5$ & $29.3 \pm 13.9$ & $3.5(0.9,6.1)$ & 0.008 \\
\hline Fat-free mass $(\mathrm{kg})$ & $62.5 \pm 14.4$ & $61.4 \pm 14.0$ & $1.8(-0.6,4.1)$ & 0.145 \\
\hline Waist (inch) & $43.1 \pm 7.0$ & $41.5 \pm 7.4$ & $1.4(0.01,2.84)$ & 0.048 \\
\hline Thigh (inch) & $25.9 \pm 3.4$ & $25.1 \pm 3.6$ & $0.7(0.02,1.44)$ & 0.044 \\
\hline Waist/thigh & $1.67 \pm 0.18$ & $1.65 \pm 0.20$ & $0.01(-0.03,0.05)$ & 0.584 \\
\hline Subjects, hyperinsulinemic, euglycemic clamp $(n)$ & 452 & 67 & & \\
\hline Resting metabolic rate (kcal/day) & $1806 \pm 338$ & $1,748 \pm 304$ & $7.5(-50.1,65.1)$ & 0.799 \\
\hline Subjects, whole-room indirect calorimetry $(n)$ & 313 & 45 & & \\
\hline 24-h energy expenditure (kcal/day) & $2,371 \pm 407$ & $2,324 \pm 365$ & $-53.4(-107.9,1.1)$ & 0.054 \\
\hline Sleeping metabolic rate (kcal/day) & $1,678 \pm 284$ & $1,616 \pm 244$ & $-23.2(-61.6,14.6)$ & 0.229 \\
\hline Subjects, ad libitum energy intake $(n)$ & 147 & 31 & & \\
\hline Carbohydrate intake (g/day) & $553 \pm 174$ & $472 \pm 139$ & $81.5(21.4,141.6)$ & 0.009 \\
\hline Fat intake (g/day) & $191 \pm 68$ & $159 \pm 54$ & $32.1(8.7,55.5)$ & 0.008 \\
\hline Protein intake (g/day) & $142 \pm 46$ & $128 \pm 41$ & $17.6(1.5,33.7)$ & $\mathbf{0 . 0 3 4}$ \\
\hline Total energy intake (kcal/day) & $4,401 \pm 1,363$ & $3,740 \pm 1,034$ & $676(190,1,162)$ & 0.007 \\
\hline Total energy intake (\% WMEN) & $157 \pm 45$ & $133 \pm 34$ & $30.9(4.2,57.6)$ & 0.02 \\
\hline
\end{tabular}

SNP rs11872292 had a low allele frequency for the A allele (mAF $=0.07)$, therefore, the heterozygous GA and homozygous AA genotypes were combined for analyses. $p$ value for percentage of body fat was adjusted for age, sex and heritage. $p$ value for total energy intake was adjusted for age, sex, heritage, fat mass and fat-free mass, and $p$ value for 24-h energy expenditure was additionally adjusted for spontaneous physical activity. All remaining $p$ values were adjusted for age, sex, percentage of body fat and heritage. Bold values indicate $p \leq 0.05$. Data are given as unadjusted mean. Estimate $(\beta)$ represents the effect per copy of the risk allele after adjusting for covariates. \% WMEN is calculated as daily energy intake/WMEN $\times 100$

$p=0.008)]$, higher waist $[\beta=1.4(0.01,2.8)$ inch, adjusted $p=0.05)]$ and thigh circumferences $[\beta=0.7(0.02,1.4)$ inch, adjusted $p=0.04]$, but not associated with the ratio of waist to thigh $(p=0.58)$ and fat-free mass $(p=0.15)$. In whole-room indirect calorimetry study $(n=358)$, the risk allele at rs11872992 was also nominally associated with a lower 24-h energy expenditure $[\beta=-53.4(-107.9,1.1)$ $\mathrm{kcal} / \mathrm{day}, p=0.054$, adjusted for age, sex, fat mass, fat-free mass, SPA and heritage]. However, there were no differences in sleeping metabolic rate $(p=0.23, n=358)$ and fasting resting metabolic rate $(p=0.80, n=519$, measured by a ventilated hood indirect calorimetry).

To test whether rs11872992 had an effect on eating behavior, data were analyzed in 178 adult participants who had a measure of ad libitum food intake assessed over 3 days and presented as the daily average. SNP rs11872992 was associated with total energy intake, where individuals with the risk allele $\mathrm{G}$ for BMI had a mean increase of total energy intake by $676(190,1,162) \mathrm{kcal} /$ day compared with the non-risk allele A carriers (Table 2, $p=0.007$, adjusted for age, sex, fat mass, fat-free mass and heritage). When energy intake was analyzed as the percentage of weight maintaining energy needs (WMEN), the $G$ allele was associated with an increase of $30.9(4.2,57.6) \%$ WMEN (adjusted $p=0.02$ ). This was reflected in a higher intake of carbohydrate [Table $2, \beta=81.5(21.4,141.6) \mathrm{g} /$ day, adjusted $p=0.007]$, fat $[\beta=32.1(8.7,55.5) \mathrm{g} / \mathrm{day}$, adjusted $p=0.008]$ and protein $[\beta=17.6(1.5,33.7) \mathrm{g} / \mathrm{day}$, adjusted $p=0.03$ ] in carriers of the $\mathrm{G}$ allele. This finding was consistent with data obtained from a separate ad libitum food intake study conducted in 55 full-heritage Pima Indian children. Those with the risk allele for rs 11872992 have a mean increase of energy intake by $504(95,913)$ $\mathrm{kcal} /$ day, or $19.2(3.6,34.7) \%$ WMEN (both $p=0.02$, adjusted for age, sex, BMI, mother's energy intake and in utero exposure to T2D). However, given the small sample size or original design of the study in children, these differences should be interpreted with caution.

Additional association analyses for all other tag SNPs

None of the individual SNPs, with the exception of rs11872992 (shown in Table 2), nor the haplotype of rs74861148 and rs483145 was associated with percentage of body fat, energy expenditure, ad libitum food intake or T2D in this study (data not shown). 


\section{Discussion}

We previously identified rare functional coding variants that cause severe early-onset monogenic forms of obesity in American Indians. However, the relative contribution of common variation in/near $M C 4 R$ to risk of obesity in this population was largely unknown. In the present study, we showed that common variation in/near MC4R has a modest effect on BMI risk in both children and adults. Although none of the common variants achieved genome-wide significance ( $p<7.2 \times 10^{-8}$, Dudbridge and Gusnanto 2008), the role of MC4R in the development of obesity is well established, thus applying such stringent criteria for statistical significance in this study may be overly conservative. Conditional analyses identified that rs 74861148 and rs483125 independently contributed to BMI risk, and an additive effect of these two SNPs on BMI was evident. Individuals with four risk alleles $(13 \%$ of the population) had an adjusted BMI that was $3.3 \mathrm{~kg} / \mathrm{m}^{2}$ greater than those with 0 or 1 risk allele (19\% of the population). In children, the adjusted $\Delta z$ score between these two groups was 0.23 , corresponding to a $\Delta \mathrm{BMI}$ of $1.4 \mathrm{~kg} / \mathrm{m}^{2}$. In comparison, children with or without rare coding functional $M C 4 R$ mutations had a $\Delta z$ score $=0.89$ (corresponding to $\Delta \mathrm{BMI}=4.8 \mathrm{~kg} / \mathrm{m}^{2}$ ) (Thearle et al. 2012). Although the effect size of common variation in $M C 4 R$ is smaller than that of the rare coding variants, because of the greater frequency/prevalence within the population, the common SNPs are likely to have a greater overall impact on obesity predisposition at the population level.

Common variants near MC4R have been reproducibly associated with BMI in GWAS of Caucasians and other populations (Loos et al. 2008; Willer et al. 2009; Speliotes et al. 2010). Several widely replicated variants in Caucasians: rs17782313, rs17700633 and rs571312 were of low frequency or rare in American Indians $(\mathrm{mAF}=0.03$, 0.005 and 0.003 , respectively), hence the statistical power was limited in this study; whereas the strongest BMI signal rs74861148 in American Indians $(\mathrm{mAF}=0.38)$ was monomorphic in Caucasians and Africans and had a $\mathrm{mAF}$ of 0.03 in Asians, indicating that American Indians may harbor a population specific BMI signal in/near MC4R. A functional promoter SNP rs11872992 had a limited statistical power due to a low minor allele frequency in Pima Indians $(\mathrm{mAF}=0.07)$, thus only achieved a nominal associations with BMI ( $p=0.05$ in adult BMI; $p=0.01$ in childhood $z$ score), despite a considerable/comparable effect size $\left(\beta=0.61 \mathrm{~kg} / \mathrm{m}^{2}\right.$ in adult BMI; 0.11 in childhood $z$ score). This SNP was also associated with obesity in a case-control study of the Belgian population $(\mathrm{mAF}=0.11$, $p=0.03$ ) (Beckers et al. 2011), but not associated with BMI in the GIANT study ( $\mathrm{mAF}=0.13, p=0.19)$, nor in a case-control study (246 obese/482 lean subjects) of the
Finnish population $(\mathrm{mAF}=0.13, p=0.57)$ (Valli-Jaakola et al. 2008). Therefore, future replications are required to further validate the significance of this SNP.

MC4R haploinsufficiency is associated with hyperphagia, binge eating, decreased energy expenditure and obesity (Farooqi et al. 2003; Branson et al. 2003). However, reports of the association of common variants in/near $M C 4 R$ with energy intake are scarce and controversial. The established variant rs17782313 near MC4R was associated with increased snacking behavior and a higher hunger score on the Three-Factor Eating Questionnaire (TFEQ) (Stutzmann et al. 2009; Stunkard and Messick 1985). An association with higher intake of total energy and dietary fat was reported (Qi et al. 2008), but could not be confirmed (Hasselbalch et al. 2010). In these studies, eating behavior traits were all assessed through self-reported questionnaires. Our study is the first to report a positive association of a common variant rs11872992 with daily food intake using an automated vending machine paradigm in a clinical research unit. At rs11872992, the BMI risk allele carriers had a substantial increase of $676 \mathrm{kcal} /$ day or $31 \%$ WMEN in total energy intake. A separate study in children provided confirmatory support. However, it is unknown why the haplotype G-A of rs74861148 and rs483145, which had the strongest association with BMI in this study $\left(p=5.5 \times 10^{-7}\right)$ was not associated with increased food intake. Although ad libitum food intake in this setting was higher than the WMEN, this system provides a more reproducible (intra-class correlation coefficient 0.9) and accurate measure of food intake than that afforded by methods based on self-report (Venti et al. 2010; Tarasuk and Beaton 1992a, b). In addition to increased food intake, the risk allele at rs11872992 was also associated with a small reduction in energy expenditure (by $53 \mathrm{kcal} / \mathrm{day}$ ) which was consistent with, although less than that observed in a prior report with the rare coding mutations in Pima Indians (by 110-140 kcal/day) (Krakoff et al. 2008).

Horstmann et al. (2013) reported that rs17782313 near $M C 4 R$ has a gender-specific impact (women only) on human brain structure and eating behavior. Consistent with this observation of a gender-specific effect near $M C 4 R$, one SNP in our study (rs62097832 in Table 1) had a genderspecific interaction $(p=0.03)$, and was associated with maximum BMI in women alone $\left(p=1 \times 10^{-5}\right)$, but not in men alone $(p=0.38)$.

SNP rs11872992 had a modest functional effect in vitro. However, we have not systematically conducted functional analyses of rs74861148 or rs483145 or variants tagged by rs483145. The ENCODE database (http://www.broadinstitute.org/mammals/haploreg) predicts that rs 74861148 may alter a regulatory motif for Hoxa5 binding, while rs 483145 could potentially affect several motifs including FOXA1, HNF4A and Pbx1, 
etc., implicating a role in transcriptional regulation. While rs74861148 is a singleton, rs483145 tags 31 variants $>55 \mathrm{~kb}$ upstream of $M C 4 R$ in Pima Indians, many of which map to transcription factor binding motifs, and two (rs10871778 and rs11661934) map to DNAse hypersensitive sites (ENCODE). If any of these variants are functional, it could explain the stronger associations with BMI observed in American Indians.

In conclusion, in this population-based study, both rare and common variants in/near $M C 4 R$ influence obesity in American Indian children and adults. We have identified a common haplotype G-A of rs74861148 and rs483145, which provides evidence for association of $M C 4 R$ with increased BMI risk in American Indians. Our longitudinal data with repeated BMI measures allowed a comprehensive assessment for the effect of MC4R on obesity risk over lifetime (age 5-50 years). Moreover, a promoter variant rs11872992 in MC4R influences risk of obesity, perhaps in part through a propensity for increased food intake and decreased energy expenditure. Our findings provide an example of the overlap in genetic determinants of monogenic and complex obesity.

Acknowledgments We thank the clinical staff of the Phoenix Epidemiology and Clinical Research Branch for conducting the study. We also thank all the participants from the Gila River Indian Community. This work was supported by the intramural research program of the National Institute of Diabetes and Digestive and Kidney Diseases, National Institutes of Health.

Conflict of interest The authors declare that there is no conflict of interest associated with this manuscript.

Open Access This article is distributed under the terms of the Creative Commons Attribution License which permits any use, distribution, and reproduction in any medium, provided the original author(s) and the source are credited.

\section{References}

Beckers S, Zegers D, de Freitas F, Mertens IL, Van Gaal LF, Van Hul W (2011) Association study of MC4R with complex obesity and replication of the rs 17782313 association signal. Mol Genet Metab 103:71-75

Bogardus C, Lillioja S, Ravussin E, Abbott W, Zawadzki JK, Young A et al (1986) Familial dependence of the resting metabolic rate. $N$ Engl J Med 315:96-100

Branson R, Potoczna N, Kral JG, Lentes KU, Hoehe MR, Horber FF (2003) Binge eating as a major phenotype of melanocortin 4 receptor gene mutations. N Engl J Med 348:1096-1103

Calton MA, Ersoy BA, Zhang S, Kane JP, Malloy MJ, Pullinger CR et al (2009) Association of functionally significant Melanocortin-4 but not Melanocortin-3 receptor mutations with severe adult obesity in a large North American case-control study. Hum Mol Genet 18:1140-1147

Dudbridge F, Gusnanto A (2008) Estimation of significance thresholds for genomewide association scans. Genet Epidemiol 32:227-234
Farooqi IS, Keogh JM, Yeo GS, Lank EJ, Cheetham T, O'Rahilly S (2003) Clinical spectrum of obesity and mutations in the melanocortin 4 receptor gene. N Engl J Med 348:1085-1095

Gluck ME, Venti CA, Lindsay RS, Knowler WC, Salbe AD, Krakoff J (2009) Maternal influence, not diabetic intrauterine environment, predicts children's energy intake. Obesity 17:772-777

Hainerová I, Larsen LH, Holst B, Finková M, Hainer V, Lebl J et al (2007) Melanocortin 4 receptor mutations in obese Czech children: studies of prevalence, phenotype development, weight reduction response, and functional analysis. J Clin Endocrinol Metab 92:3689-3696

Hanis CL, Chakraborty R, Ferrell RE, Schull WJ (1986) Individual admixture estimates: disease associations and individual risk of diabetes and gallbladder disease among Mexican-Americans in Starr County, Texas. Am J Phys Anthropol 170:433-441

Hasselbalch AL, Angquist L, Christiansen L, Heitmann BL, Kyvik KO, Sørensen TI (2010) A variant in the fat mass and obesityassociated gene (FTO) and variants near the melanocortin-4 receptor gene (MC4R) do not influence dietary intake. J Nutr 140:831-834

Hinney A, Bettecken T, Tarnow P, Brumm H, Reichwald K, Lichtner $P$ et al (2006) Prevalence, spectrum, and functional characterization of melanocortin-4 receptor gene mutations in a representative population-based sample and obese adults from Germany. Clin Endocrinol Metab 91:1761-1769

Horstmann A, Kovacs P, Kabisch S, Boettcher Y, Schloegl H, Tönjes A et al (2013) Common genetic variation near MC4R has a sexspecific impact on human brain structure and eating behavior. PLoS One 8:e74362

Knowler WC, Pettitt DJ, Saad MF, Bennett PH (1990) Diabetes mellitus in the Pima Indians: incidence, risk factors and pathogenesis. Diabetes Metab Rev 6:1-27

Krakoff J, Ma L, Kobes S, Knowler WC, Hanson RL, Bogardus C et al (2008) Lower metabolic rate in individuals heterozygous for either a frameshift or a functional missense MC4R variant. Diabetes $57: 3267-3272$

Lillioja S, Mott DM, Spraul M, Ferraro R, Foley JE, Ravussin E et al (1993) Insulin resistance and insulin secretory dysfunction as precursors of non-insulin-dependent diabetes mellitus. Prospective studies of Pima Indians. N Engl J Med 329:1988-1992

Loos RJ, Lindgren CM, Li S, Wheeler E, Zhao JH, Prokopenko I et al (2008) Common variants near MC4R are associated with fat mass, weight and risk of obesity. Nat Genet 40:768-775

Ma L, Hanson RL, Traurig MT, Muller YL, Kaur BP, Perez JM et al (2010) Evaluation of A2BP1 as an obesity gene. Diabetes 59:2837-2845

Muller YL, Bogardus C, Beamer BA, Shuldiner AR, Baier LJ (2003) A functional variant in the peroxisome proliferator-activated receptor gamma 2 promoter is associated with predictors of obesity and type 2 diabetes in Pima Indians. Diabetes 52:1864-1871

Muller YL, Piaggi P, Hoffman D, Huang K, Gene B, Kobes S et al (2014) Common genetic variation in the glucokinase gene (GCK) is associated with type 2 diabetes and rates of carbohydrate oxidation and energy expenditure. Diabetologia 57:1382-1390

Pannacciulli N, Salbe AD, Ortega E, Venti CA, Bogardus C, Krakoff $\mathrm{J}$ (2007) The 24-h carbohydrate oxidation rate in a human respiratory chamber predicts ad libitum food intake. Am J Clin Nutr 86:625-632

Piaggi P, Thearle MS, Bogardus C, Krakoff J (2013a) Lower energy expenditure predicts long-term increases in weight and fat mass. $\mathbf{J}$ Clin Endocrinol Metab 98:E703-E707

Piaggi P, Krakoff J, Bogardus C, Thearle MS (2013b) Lower 'awake and fed thermogenesis' predicts future weight gain in subjects with abdominal adiposity. Diabetes 62:4043-4051

Qi L, Kraft P, Hunter DJ, Hu FB (2008) The common obesity variant near MC4R gene is associated with higher intakes of total energy 
and dietary fat, weight change and diabetes risk in women. Hum Mol Genet 17:3502-3508

Ravussin E, Lillioja S, Anderson TE, Christin L, Bogardus C (1986) Determinants of 24-h energy expenditure in man. Methods and results using a respiratory chamber. J Clin Invest 78:1568-1578

Speliotes EK, Willer CJ, Berndt SI, Monda KL, Thorleifsson G, Jackson AU et al (2010) Association analyses of 249,796 individuals reveal 18 new loci associated with body mass index. Nat Genet 42:937-948

Stunkard AJ, Messick S (1985) The three-factor eating questionnaire to measure dietary restraint, disinhibition and hunger. J Psychosom Res 29:71-83

Stutzmann F, Cauchi S, Durand E, Calvacanti-Proença C, Pigeyre M, Hartikainen AL et al (2009) Common genetic variation near MC4R is associated with eating behaviour patterns in European populations. Int J Obes 33:373-378

Tarasuk V, Beaton GH (1992a) Statistical estimation of dietary parameters: implications of patterns in within-subject variation-a case study of sampling strategies. Am J Clin Nutr 55:22-27

Tarasuk V, Beaton GH (1992b) Day-to-day variation in energy and nutrient intake: evidence of individuality in eating behaviour? Appetite 18:43-54

Tataranni PA, Ravussin E (1995) Use of dual-energy X-ray absorptiometry in obese individuals. Am J Clin Nutr 62:730-734

The Expert Committee on the Diagnosis and Classification of Diabetes Mellitus (1997) Report of the Expert Committee on the
Diagnosis and Classification of Diabetes Mellitus. Diabetes Care 20:1183-1197

Thearle MS, Muller YL, Hanson RL, Mullins M, Abdussamad M, Tran J et al (2012) Greater impact of melanocortin-4 receptor deficiency on rates of growth and risk of type 2 diabetes during childhood compared with adulthood in Pima Indians. Diabetes 61:250-257

Tian C, Hinds DA, Shigeta R, Adler SG, Lee A, Pahl MV et al (2007) A genomewide single-nucleotide-polymorphism panel for Mexican American admixture mapping. Am J Hum Genet 80:1014-1023

Valli-Jaakola K, Suviolahti E, Schalin-Jäntti C, Ripatti S, Silander K, Oksanen L et al (2008) Further evidence for the role of ENPP1 in obesity: association with morbid obesity in Finns. Obesity 16:2113-2119

Venti CA, Votruba SB, Franks PW, Krakoff J, Salbe AD (2010) Reproducibility of ad libitum energy intake with the use of a computerized vending machine system. Am J Clin Nutr 91:343-348

Vozarova de Courten B, Hanson RL, Funahashi T, Lindsay RS, Matsuzawa Y, Tanaka $S$ et al (2005) Common polymorphisms in the Adiponectin Gene ACDC are not associated with diabetes in Pima Indians. Diabetes 54:284-289

Willer CJ, Speliotes EK, Loos RJ, Li S, Lindgren CM, Heid IM et al (2009) Six new loci associated with body mass index highlight a neuronal influence on body weight regulation. Nat Genet 41:25-34 